CRYSTALLOGRAPHIC COMMUNICATIONS

ISSN 2056-9890

Received 4 March 2019

Accepted 15 March 2019

Edited by H. Ishida, Okayama University, Japan

Keywords: crystal structure; 4-chlorophenyl; 4-fluorophenyl; face-to-face $\pi$ - $\pi$ stacking interaction; Hirshfeld surface analysis.

CCDC reference: 1882554

Supporting information: this article has supporting information at journals.iucr.org/e

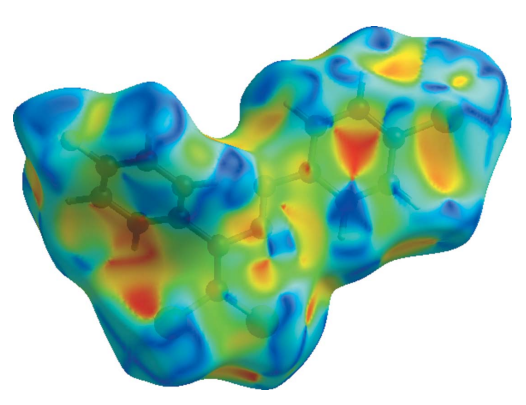

OPEN $\odot$ ACCESS

\section{Crystal structure and Hirshfeld surface analysis of (E)-1-(4-chlorophenyl)-2-[2,2-dichloro-1-(4-fluoro- phenyl)ethenyl]diazene}

\author{
Namiq Q. Shikhaliyev, ${ }^{a}$ Sevim Türktekin Celikesir, ${ }^{\mathrm{b}}$ Mehmet Akkurt, ${ }^{\mathrm{b}}$ Khanim N. \\ Bagirova, ${ }^{a}$ Gulnar T. Suleymanova ${ }^{a}$ and Flavien A. A. Toze ${ }^{c_{*}}$
}

aOOrganic Chemistry Department, Baku State University, Z. Xalilov str. 23, Az, 1148 Baku, Azerbaijan, ${ }^{\mathbf{b}}$ Department of Physics, Faculty of Sciences, Erciyes University, 38039 Kayseri, Turkey, and ${ }^{\mathrm{C}}$ Department of Chemistry, Faculty of Sciences, University of Douala, PO Box 24157, Douala, Republic of Cameroon. *Correspondence e-mail: toflavien@yahoo.fr

In the title compound, $\mathrm{C}_{14} \mathrm{H}_{8} \mathrm{Cl}_{3} \mathrm{FN}_{2}$, the planes of the 4-fluorophenyl ring and the 4-chlorophenyl ring make a dihedral angle of $56.13(13)^{\circ}$. In the crystal, molecules are stacked in a column along the $a$ axis via a weak $\mathrm{C}-\mathrm{H} \cdots \mathrm{Cl}$ hydrogen bond and face-to-face $\pi-\pi$ stacking interactions [centroid-centroid distances $=3.8615(18)$ and $3.8619(18) \AA$ ] . The crystal packing is further stabilized by short $\mathrm{Cl} \cdots \mathrm{Cl}$ contacts. The Hirshfeld surface analysis of the crystal structure indicates that the most important contributions for the crystal packing are from $\mathrm{Cl} \cdots \mathrm{H} / \mathrm{H} \cdots \mathrm{Cl}(31.2 \%), \mathrm{H} \cdots \mathrm{H}(14.8 \%), \mathrm{C} \cdots \mathrm{H} / \mathrm{H} \cdots \mathrm{C}(14.0 \%), \mathrm{F} \cdots \mathrm{H} /$ $\mathrm{H} \cdots \mathrm{F}(12.8 \%), \mathrm{C} \cdots \mathrm{C}(9.0 \%)$ and $\mathrm{Cl} \cdots \mathrm{Cl}(6.7 \%)$ interactions.

\section{Chemical context}

Azo compounds provide ubiquitous motifs in synthetic chemistry and are widely used as organic dyes, indicators, molecular switches, pigments, ligands, food additives, radical reaction initiators, therapeutic agents etc. (Gurbanov et al., 2017; Maharramov et al., 2018; Mahmudov et al., 2019). Azo dyes are also convenient model compounds to study both $E / Z$ isomerization and noncovalent interactions (Mahmudov et al., 2015; Shixaliyev et al., 2018). Thus, decorating the structure of dyes with tailored functionalities (noncovalent bond donor centres) can be a pivotal strategy for controlling and tuning their functional properties (Mahmudov et al., 2017; Zubkov et al., 2018). Herein we report the molecular structure and noncovalent interactions in the title compound.<smiles>Fc1ccc(C(/N=N/c2ccc(Cl)cc2)=C(Cl)Cl)cc1</smiles>

\section{Structural commentary}

The molecular conformation of the title compound is not planar (Fig. 1); the planes of the 4-fluorophenyl ring and the 4-chlorophenyl ring form a dihedral angle of $56.13(13)^{\circ}$. The $\mathrm{C} 4-\mathrm{C} 3-\mathrm{C} 1-\mathrm{N} 1, \quad \mathrm{C} 8-\mathrm{C} 3-\mathrm{C} 1-\mathrm{C} 2, \quad \mathrm{C} 3-\mathrm{C} 1-\mathrm{C} 2-\mathrm{Cl} 1$, $\mathrm{C} 3-\mathrm{C} 1-\mathrm{C} 2-\mathrm{Cl} 2, \mathrm{~N} 1-\mathrm{C} 1-\mathrm{C} 2-\mathrm{Cl} 1, \mathrm{~N} 1-\mathrm{C} 1-\mathrm{C} 2-\mathrm{Cl}$, $\mathrm{C} 1-\mathrm{N} 1-\mathrm{N} 2-\mathrm{C} 9$ and $\mathrm{N} 1-\mathrm{N} 2-\mathrm{C} 9-\mathrm{C} 14$ torsion angles are 48.4 (4), 49.2 (4), -1.9 (4), 177.94 (19), 177.14 (18), -3.0 (3), $179.2(2)$ and $175.9(2)^{\circ}$, respectively. 


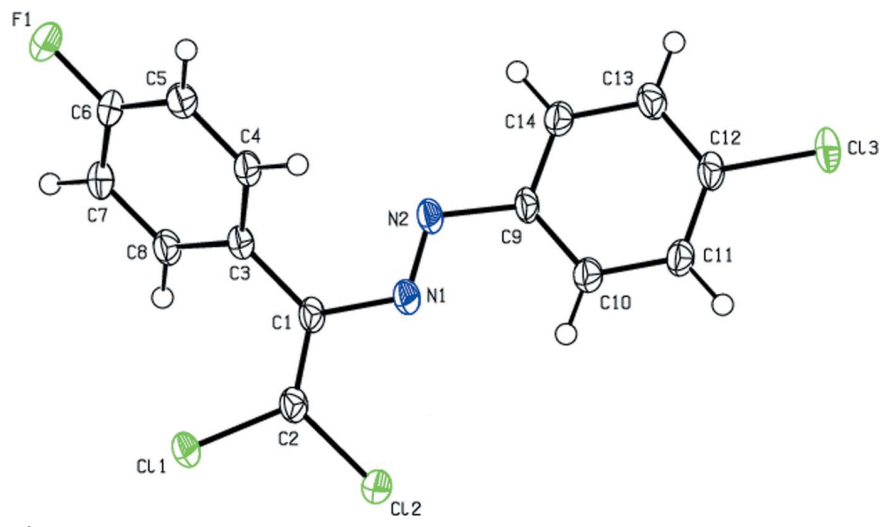

Figure 1

The molecular structure of the title compound, with the atom-labelling scheme and $50 \%$ probability displacement ellipsoids.

\section{Supramolecular features and Hirshfeld surface analysis}

In the crystal, molecules are linked by a weak $\mathrm{C}-\mathrm{H} \cdots \mathrm{Cl}$ hydrogen bond (Table 1), forming a column along the $a$ axis (Figs. 2 and 3). The column is further stabilized by face-to-face $\pi-\pi$ stacking interactions; the centroid-centroid distances between the adjacent $\mathrm{C} 3-\mathrm{C} 8$ rings and between the adjacent C9-C14 rings are 3.8615 (18) and 3.8619 (18) A, respectively. Moreover, the columns are linked by intermolecular $\mathrm{Cl} \cdots \mathrm{Cl}$ short contacts, with distances of 3.3756 (11) and 3.3841 (11) $\AA$ (Table 2), forming a layer parallel to the $b c$ plane (Fig. 2).

Hirshfeld surfaces and fingerprint plots were generated for the title compound using CrystalExplorer (McKinnon et al., 2007). The Hirshfeld surface mapped over $d_{\text {norm }}$ using a standard surface resolution with a fixed colour scale of -0.0941 (red) to 1.4174 a.u. (blue) is shown in Fig. 4. This plot was generated to quantify and visualize the intermolecular interactions and to explain the observed crystal packing. The dark-red spots on the $d_{\text {norm }}$ surface arise as a result of the $\mathrm{C}-$ $\mathrm{H} \cdots \mathrm{Cl}$ interaction and short interatomic contacts (Tables 1 and 2), while the other weaker intermolecular interactions appear as light-red spots. The shape index of the Hirshfeld

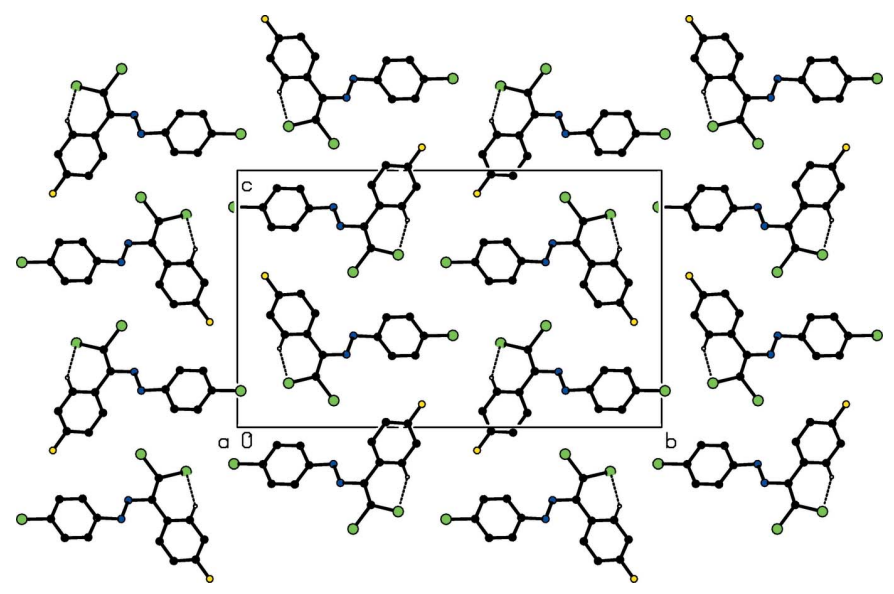

Figure 2

A packing diagram of the title compound, viewed along the $a$ axis, showing the $\mathrm{C}-\mathrm{H} \cdots \mathrm{Cl}$ interactions (dashed lines).
Table 1

Hydrogen-bond geometry $\left(\AA{ }^{\circ}\right)$.

\begin{tabular}{lllll}
\hline$D-\mathrm{H} \cdots A$ & $D-\mathrm{H}$ & $\mathrm{H} \cdots A$ & $D \cdots A$ & $D-\mathrm{H} \cdots A$ \\
\hline $\mathrm{C} 8-\mathrm{H} 8 \cdots \mathrm{Cl}{ }^{\mathrm{i}}$ & 0.95 & 2.81 & $3.634(3)$ & 146 \\
\hline
\end{tabular}

Symmetry code: (i) $x-1, y, z$.

Table 2

Summary of short interatomic contacts $(\AA)$ in the title compound.

\begin{tabular}{lll}
\hline Contact & Distance & Symmetry operation \\
\hline $\mathrm{H} 4 \cdots \mathrm{N} 2$ & 2.67 & $1+x, y, z$ \\
$\mathrm{C} 1 \cdots \mathrm{Cl} 3$ & $3.3756(11)$ & $-x,-\frac{1}{2}+y, \frac{1}{2}-z$ \\
$\mathrm{Cl} \cdots \mathrm{Cl} 3$ & $3.3841(11)$ & $1-x,-\frac{1}{2}+y, \frac{1}{2}-z$ \\
$\mathrm{C} 2 \cdots \mathrm{H} 14$ & 3.03 & $1+x, \frac{1}{2}-y,-\frac{1}{2}+z$ \\
$\mathrm{H} 11 \cdots \mathrm{F} 1$ & 2.81 & $x, \frac{1}{2}-y,-\frac{1}{2}+z$ \\
$\mathrm{H} 7 \cdots \mathrm{F} 1$ & 2.67 & $1-x,-y, 1-z$ \\
$\mathrm{~F} 1 \cdots \mathrm{H} 11$ & 2.84 & $1+x, \frac{1}{2}-y, \frac{1}{2}+z$ \\
\hline
\end{tabular}

surface is a tool to visualize the $\pi-\pi$ stacking by the presence of adjacent red and blue triangles; if there are no adjacent red and/or blue triangles, then there are no $\pi-\pi$ interactions. Fig. 5 clearly suggests that there are $\pi-\pi$ interactions in the title compound.

The percentage contributions of the various contacts to the total Hirshfeld surface are shown in the 2D fingerprint plots in Fig. 6. The reciprocal $\mathrm{Cl} \cdots \mathrm{H} / \mathrm{H} \cdots \mathrm{Cl}$ interactions appear as two symmetrical broad wings with $d_{\mathrm{e}}+d_{\mathrm{i}} \simeq 2.7 \AA$ and contribute $31.2 \%$ to the Hirshfeld surface (Fig. $6 b$ ). The $\mathrm{H} \cdots \mathrm{H}$ interactions appear in the middle of the scattered

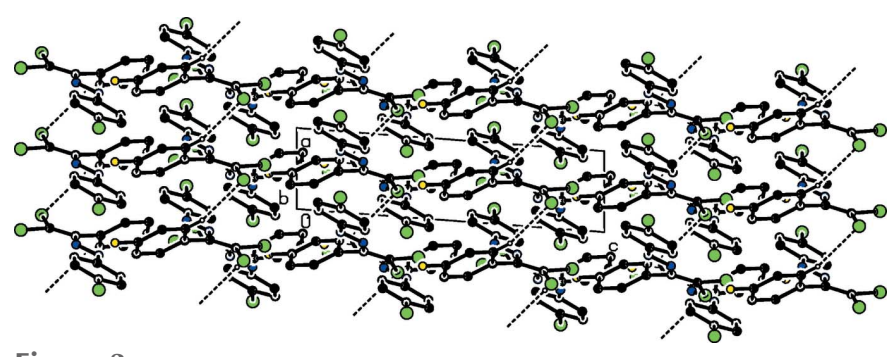

Figure 3

A packing diagram of the title compound, viewed along the $b$ axis, showing the $\mathrm{C}-\mathrm{H} \cdots \mathrm{Cl}$ interactions (dashed lines).

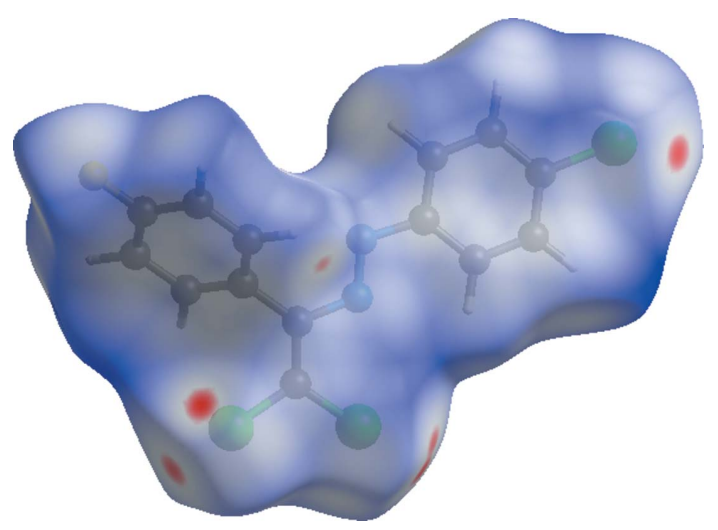

Figure 4

View of the Hirshfeld surface of the title compound plotted over $d_{\text {norm }}$ in the range from -0.0941 to 1.4174 a.u. 
Figure 5

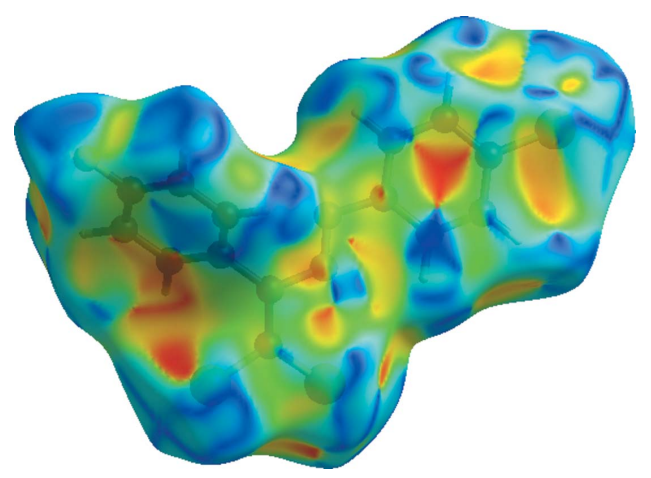

View of the Hirshfeld surface of the title compound plotted over shape index.

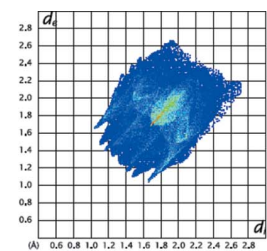

(a) All...All

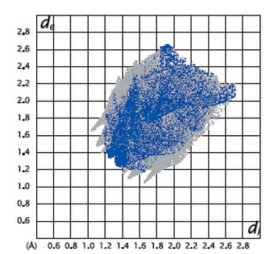

(c) $\mathrm{H} . . . \mathrm{H}$

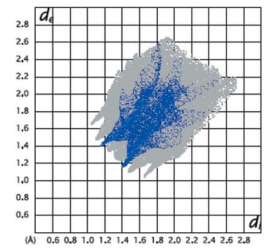

(e) $\mathrm{F} \ldots \mathrm{H} / \mathrm{H} \ldots \mathrm{F}$

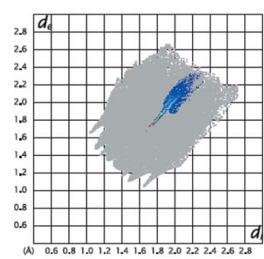

(g) $\mathrm{Cl}$...Cl

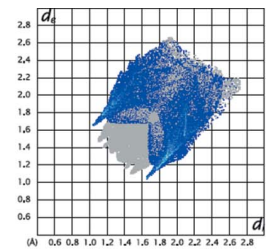

(b) $\mathrm{Cl} \ldots \mathrm{H} / \mathrm{H} \ldots \mathrm{Cl}$

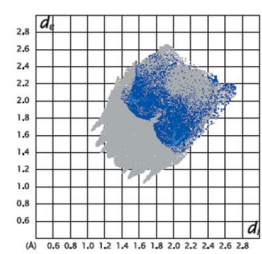

(d) $\mathrm{C} . . \mathrm{H} / \mathrm{H}$...C

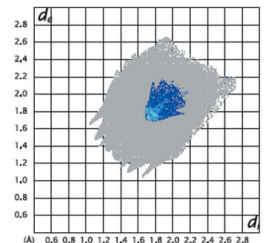

(f) $\mathrm{C}$...C

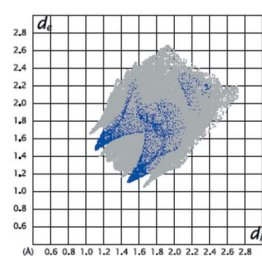

(h) $\mathbf{N} \ldots . . H / H \ldots N$

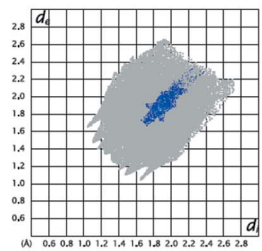

(i) $\mathrm{Cl} \ldots \mathrm{C} / \mathrm{C} \ldots \mathrm{Cl}$

Figure 6

The full 2D fingerprint plots for the title compound, showing $(a)$ all interactions, and those delineated into $(b) \mathrm{Cl} \cdots \mathrm{H} / \mathrm{H} \cdots \mathrm{Cl},(c) \mathrm{H} \cdots \mathrm{H},(d)$ $\mathrm{C} \cdots \mathrm{H} / \mathrm{H} \cdots \mathrm{C},(e) \mathrm{F} \cdots \mathrm{H} / \mathrm{H} \cdots \mathrm{F},(f) \mathrm{C} \cdots \mathrm{C},(g) \mathrm{Cl} \cdots \mathrm{Cl},(h) \mathrm{N} \cdots \mathrm{H} / \mathrm{H} \cdots \mathrm{N}$ and $(i) \mathrm{Cl} \cdots \mathrm{C} / \mathrm{C} \cdots \mathrm{Cl}$ interactions. The $d_{\mathrm{i}}$ and $d_{\mathrm{e}}$ values are the closest internal and external distances (in $\AA$ ) from given points on the Hirshfeld surface contacts. points in the 2D fingerprint plots, with an overall contribution to the Hirshfeld surface of $14.8 \%$ (Fig. $6 c$ ). The $\mathrm{C} \cdots \mathrm{H} / \mathrm{H} \cdots \mathrm{C}$ interactions, with a $14.0 \%$ contribution, are present as bump symmetrical spikes at diagonal axes (Fig. $6 d$ ). The F..H/ $\mathrm{H} \cdots \mathrm{F}$ interactions, with a $12.8 \%$ contribution, are present as sharp symmetrical spikes at diagonal axes $d_{\mathrm{e}}+d_{\mathrm{i}} \simeq 2.55 \AA$ (Fig. 6e). The C $\cdots \mathrm{C}$ interactions appear in the middle of the scattered points in the 2D fingerprint plots with an overall contribution to the Hirshfeld surface of $9.0 \%$ (Fig. $6 f$ ). The small percentage contributions from the other different interatomic contacts to the Hirshfeld surfaces are as follows:

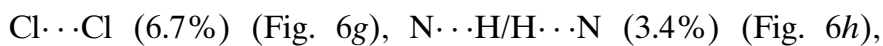
$\mathrm{Cl} \cdots \mathrm{C} / \mathrm{C} \cdots \mathrm{Cl}(3.1 \%)$ (Fig. $6 i), \mathrm{N} \cdots \mathrm{C} / \mathrm{C} \cdots \mathrm{N}(2.8 \%), \mathrm{N} \cdots \mathrm{N}$ $(1.0 \%), \mathrm{Cl} \cdots \mathrm{N} / \mathrm{N} \cdots \mathrm{Cl}(0.8 \%), \mathrm{F} \cdots \mathrm{F}(0.4 \%)$ and $\mathrm{F} \cdots \mathrm{C} / \mathrm{C} \cdots \mathrm{F}$ $(0.1 \%)$. Hirshfeld surface representations with the function $d_{\text {norm }}$ plotted onto the surface for $\mathrm{Cl} \cdots \mathrm{H} / \mathrm{H} \cdots \mathrm{Cl}, \mathrm{H} \cdots \mathrm{H}$,

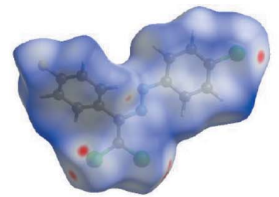

(a) All...All

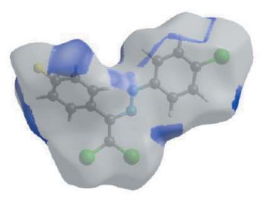

(c) H...H

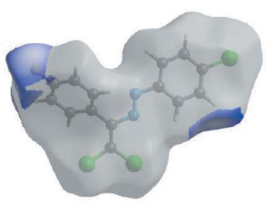

(e) F...H/H...F

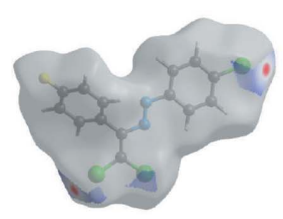

(g) Cl...Cl

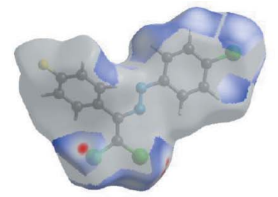

(b) $\mathrm{Cl} \cdots \mathbf{H} / \mathrm{H} \cdots \mathrm{Cl}$

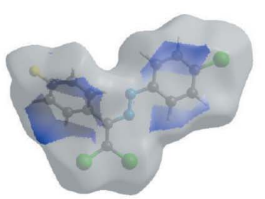

(d) $\mathrm{C} \ldots . \mathrm{H} / \mathrm{H} \ldots \mathrm{C}$

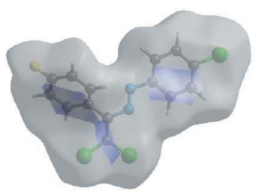

(f) $\mathrm{C} \ldots \mathrm{C}$

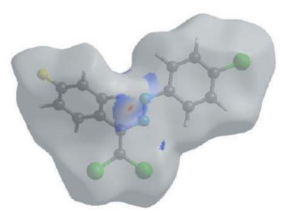

(h) $\mathbf{N}$...H/H...N

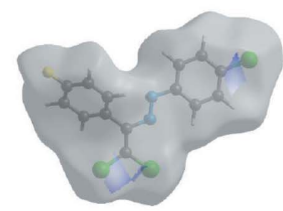

Figure 7

(i) $\mathrm{Cl} . . \mathrm{C} / \mathrm{C} \ldots \mathrm{Cl}$

Hirshfeld surface representations with the function $d_{\text {norm }}$ plotted onto the surface for $(a)$ all interactions, $(b) \mathrm{Cl} \cdots \mathrm{H} / \mathrm{H} \cdots \mathrm{Cl},(c) \mathrm{H} \cdots \mathrm{H},(d) \mathrm{C} \cdot \cdots \mathrm{H} /$ $\mathrm{H} \cdots \mathrm{C},(e) \mathrm{F} \cdots \mathrm{H} / \mathrm{H} \cdots \mathrm{F},(f) \mathrm{C} \cdots \mathrm{C},(g) \mathrm{Cl} \cdots \mathrm{Cl},(h) \mathrm{N} \cdots \mathrm{H} / \mathrm{H} \cdots \mathrm{N}$ and $(i)$ $\mathrm{Cl} \cdots \mathrm{C} / \mathrm{C} \cdots \mathrm{Cl}$ interactions. 
$\mathrm{C} \cdots \mathrm{H} / \mathrm{H} \cdots \mathrm{C}, \mathrm{F} \cdots \mathrm{H} / \mathrm{H} \cdots \mathrm{F}, \mathrm{C} \cdots \mathrm{C}, \mathrm{Cl} \cdots \mathrm{Cl}, \mathrm{N} \cdots \mathrm{H} / \mathrm{H} \cdots \mathrm{N}$ and $\mathrm{Cl} \cdots \mathrm{C} / \mathrm{C} \cdots \mathrm{Cl}$ interactions are shown in Fig. 7. The large number of $\mathrm{Cl} \cdots \mathrm{H} / \mathrm{H} \cdots \mathrm{Cl}, \mathrm{H} \cdots \mathrm{H}, \mathrm{C} \cdots \mathrm{H} / \mathrm{H} \cdots \mathrm{C}, \mathrm{F} \cdots \mathrm{H} / \mathrm{H} \cdots \mathrm{F}$ and $\mathrm{C} \cdots \mathrm{C}$ interactions suggest that van der Waals interactions and hydrogen bonding play the major roles in the crystal packing (Hathwar et al., 2015).

\section{Database survey}

A search of the Cambridge Structural Database (CSD, Version 5.40, November 2018; Groom et al., 2016) for structures having an (E)-1-(2,2-dichloro-1-phenylvinyl)-2-phenyldiazene unit gave 18 hits. Three compounds closely resemble the title compound, viz. 1-[2,2-dichloro-1-(4-nitrophenyl)ethenyl]-2-(4-fluorophenyl)diazene (CSD refcode XIZREG; Atioğlu et al., 2019), 1,1'-[methylenebis(4,1phenylene)]bis[(2,2-dichloro-1-(4-nitrophenyl)ethenyl]diazene (LEQXIR; Shixaliyev et al., 2018) and 1,1'-[methylenebis(4,1phenylene)]bis $\{[2,2$-dichloro-1-(4-chlorophenyl)ethenyl]diazene $\}$ (LEQXOX; Shixaliyev et al., 2018). In XIZREG (Atioğlu et al., 2019), molecules are linked by a C-H. . O hydrogen bond into a zigzag chain running along the $c$ axis. The crystal packing is further stabilized by $\mathrm{C}-\mathrm{Cl} \cdots \pi, \mathrm{C}-\mathrm{F} \cdots \pi$ and $\mathrm{N}-\mathrm{O} \cdots \pi$ interactions. In the crystal of LEQXIR, $\mathrm{C}-\mathrm{H} \cdots \mathrm{N}$ and $\mathrm{C}-$ $\mathrm{H} \cdot \cdots \mathrm{O}$ hydrogen bonds and $\mathrm{Cl} \cdots \mathrm{O}$ contacts were found, and in LEQXOX, C-H. $\cdots \mathrm{N}$ and $\mathrm{Cl} \cdots \mathrm{Cl}$ contacts were observed.

\section{Synthesis and crystallization}

This dye was synthesized according to a reported method (Shixaliyev et al., 2018). A $20 \mathrm{ml}$ screw-necked vial was charged with dimethyl sulfoxide $(10 \mathrm{ml}),(E)-1$-(4-chlorophenyl)-2-(4-fluorobenzylidene)hydrazine (248 mg, $1 \mathrm{mmol}$ ), tetramethylethylenediamine $(295 \mathrm{mg}, 2.5 \mathrm{mmol}), \mathrm{CuCl}(2 \mathrm{mg}$, $0.02 \mathrm{mmol})$ and $\mathrm{CCl}_{4}(20 \mathrm{mmol}, 10$ equiv.). After 1-3 h (until thin-layer chromatography analysis showed complete consumption of the corresponding Schiff base), the reaction mixture was poured into a $\sim 0.01 M$ solution of $\mathrm{HCl}(100 \mathrm{ml}$, $\sim \mathrm{pH}=2-3)$ and extracted with dichloromethane $(3 \times 20 \mathrm{ml})$. The combined organic phase was washed with water $(3 \times$ $50 \mathrm{ml})$, brine $(30 \mathrm{ml})$, dried over anhydrous $\mathrm{Na}_{2} \mathrm{SO}_{4}$ and concentrated in vacuo with a rotary evaporator. The residue was purified by column chromatography on silica gel using appropriate mixtures of hexane and dichloromethane (3:1-1:1 $v / v)$.

Red solid (yield 46\%); m.p. 340-338 K. Analysis calculated (\%) for $\mathrm{C}_{14} \mathrm{H}_{8} \mathrm{Cl}_{3} \mathrm{FN}_{2}$ : C 51.02, $\mathrm{H} 2.45, \mathrm{~N}$ 8.50; found: $\mathrm{C} 49.95$, $\mathrm{H} 2.43, \mathrm{~N}$ 8.47. ${ }^{1} \mathrm{H}$ NMR $\left(300 \mathrm{MHz}, \mathrm{CDCl}_{3}\right): \delta$ 7.15-7.17 $(\mathrm{m}$, $4 \mathrm{H}), 7.42-7.45(d, 2 \mathrm{H}, J=9.21 \mathrm{~Hz}), 7.73-7.75(d, 2 \mathrm{H}, J=$ $6.04 \mathrm{~Hz}) .{ }^{13} \mathrm{C} \mathrm{NMR}\left(75 \mathrm{MHz}, \mathrm{CDCl}_{3}\right): \delta 115.29,115.58,124.49$, 127.46, 129.37, 130.43, 131.88, 131.99, 137.73, 151.13. ESI-MS: $m / z: 330.44[M+\mathrm{H}]^{+}$.

\section{Refinement}

Crystal data, data collection and structure refinement details are summarized in Table 3. C-bound $\mathrm{H}$ atoms were
Table 3

Experimental details.

\begin{tabular}{ll}
\hline Crystal data & \\
Chemical formula & $\mathrm{C}_{14} \mathrm{H}_{8} \mathrm{Cl}_{3} \mathrm{FN}_{2}$ \\
$M_{\mathrm{r}}$ & 329.57 \\
Crystal system, space group & Monoclinic, $P 2_{1} / c$ \\
Temperature $(\mathrm{K})$ & 100 \\
$a, b, c(\AA)$ & $3.8617(8), 24.249(5), 14.724(3)$ \\
$\beta\left({ }^{\circ}\right)$ & $94.30(3)$ \\
$V\left(\AA^{3}\right)$ & $1374.9(5)$ \\
$Z$ & 4 \\
Radiation type & Synchrotron, $\lambda=0.80246 \AA$ \\
$\mu\left(\mathrm{mm}^{-1}\right)$ & 0.93 \\
Crystal size $(\mathrm{mm})$ & $0.20 \times 0.10 \times 0.02$ \\
& \\
Data collection & Rayonix SX165 CCD \\
Diffractometer & Multi-scan $(S C A L A ;$ Evans, 2006) \\
Absorption correction & $0.840,0.970$ \\
$T_{\min }, T_{\text {max }}$ & $20761,2984,2719$ \\
No. of measured, independent and & \\
$\quad$ observed $[I>2 \sigma(I)]$ reflections & 0.115 \\
$R_{\text {int }}$ & 0.640 \\
$(\text { sin } \theta / \lambda)_{\text {max }}\left(\AA^{-1}\right)$ & \\
& \\
Refinement & \\
$R\left[F^{2}>2 \sigma\left(F^{2}\right)\right], w R\left(F^{2}\right), S$ & $0.053,0.142,1.05$ \\
No. of reflections & 2984 \\
No. of parameters & 182 \\
H-atom treatment & $\mathrm{H}$-atom parameters constrained \\
$\Delta \rho_{\text {max }}, \Delta \rho_{\text {min }}\left(\mathrm{e} \AA^{-3}\right)$ & $0.59,-0.72$ \\
\hline
\end{tabular}

Computer programs: Marccd (Doyle, 2011), iMosflm (Battye et al., 2011), SHELXS97 (Sheldrick, 2008), SHELXL2018 (Sheldrick, 2015), ORTEP-3 for Windows (Farrugia, 2012) and PLATON (Spek, 2009).

constrained to an ideal geometry, with $\mathrm{C}-\mathrm{H}=0.95 \AA$ and $U_{\text {iso }}(\mathrm{H})=1.2 U_{\text {eq }}(\mathrm{C})$. Nine outliers $(\overline{4}, 2,12 ; \overline{4}, 1,12 ; \overline{3}, 18,11$; $2,21,1 ; \overline{4}, 3,12 ; \overline{3}, 19,10 ; 0,13,17 ; \overline{4}, 4,10 ; 2,20,0)$ were omitted in the final cycles of refinement.

\section{Funding information}

Funding for this research was provided by: Science Development Foundation under the President of the Republic of Azerbaijan (grant No. EIF/MQM/Elm-Tehsil-1-2016-1(26)-71/ 06/4).

\section{References}

Atioğlu, Z., Akkurt, M., Shikhaliyev, N. Q., Suleymanova, G. T., Bagirova, K. N. \& Toze, F. A. A. (2019). Acta Cryst. E75, $237-$ 241.

Battye, T. G. G., Kontogiannis, L., Johnson, O., Powell, H. R. \& Leslie, A. G. W. (2011). Acta Cryst. D67, 271-281.

Doyle, R. A. (2011). Marccd software manual. Rayonix LLC, Evanston, USA.

Evans, P. (2006). Acta Cryst. D62, 72-82.

Farrugia, L. J. (2012). J. Appl. Cryst. 45, 849-854.

Groom, C. R., Bruno, I. J., Lightfoot, M. P. \& Ward, S. C. (2016). Acta Cryst. B72, 171-179.

Gurbanov, A. V., Mahmudov, K. T., Kopylovich, M. N., Guedes da Silva, M. F. C., Sutradhar, M., Guseinov, F. I., Zubkov, F. I., Maharramov, A. M. \& Pombeiro, A. J. L. (2017). Dyes Pigments, 138, 107-111.

Hathwar, V. R., Sist, M., Jørgensen, M. R. V., Mamakhel, A. H., Wang, X., Hoffmann, C. M., Sugimoto, K., Overgaard, J. \& Iversen, B. B. (2015). IUCrJ, 2, 563-574. 
Maharramov, A. M., Shikhaliyev, N. Q., Suleymanova, G. T., Gurbanov, A. V., Babayeva, G. V., Mammadova, G. Z., Zubkov, F. I., Nenajdenko, V. G., Mahmudov, K. T. \& Pombeiro, A. J. L. (2018). Dyes Pigments, 159, 135-141.

Mahmudov, K. T., Guedes da Silva, M. F. C., Sutradhar, M., Kopylovich, M. N., Huseynov, F. E., Shamilov, N. T., Voronina, A. A., Buslaeva, T. M. \& Pombeiro, A. J. L. (2015). Dalton Trans. 44, 5602-5610.

Mahmudov, K. T., Gurbanov, A. V., Guseinov, F. I. \& Guedes da Silva, M. F. C. (2019). Coord. Chem. Rev. 387, 32-46.

Mahmudov, K. T., Kopylovich, M. N., Guedes da Silva, M. F. C. \& Pombeiro, A. J. L. (2017). Coord. Chem. Rev. 345, 54-72.
McKinnon, J. J., Jayatilaka, D. \& Spackman, M. A. (2007). Chem. Commun. pp. 3814-3816.

Sheldrick, G. M. (2008). Acta Cryst. A64, 112-122.

Sheldrick, G. M. (2015). Acta Cryst. C71, 3-8.

Shixaliyev, N. Q., Ahmadova, N. E., Gurbanov, A. V., Maharramov, A. M., Mammadova, G. Z., Nenajdenko, V. G., Zubkov, F. I., Mahmudov, K. T. \& Pombeiro, A. J. L. (2018). Dyes Pigments, 150, 377-381.

Spek, A. L. (2009). Acta Cryst. D65, 148-155.

Zubkov, F. I., Mertsalov, D. F., Zaytsev, V. P., Varlamov, A. V., Gurbanov, A. V., Dorovatovskii, P. V., Timofeeva, T. V., Khrustalev, V. N. \& Mahmudov, K. T. (2018). J. Mol. Liq. 249, 949-952. 


\section{supporting information}

Acta Cryst. (2019). E75, 465-469 [https://doi.org/10.1107/S2056989019003657]

\section{Crystal structure and Hirshfeld surface analysis of (E)-1-(4-chlorophenyl)-2-[2,2- dichloro-1-(4-fluorophenyl)ethenyl]diazene}

Namiq Q. Shikhaliyev, Sevim Türktekin Çelikesir, Mehmet Akkurt, Khanim N. Bagirova, Gulnar

\section{T. Suleymanova and Flavien A. A. Toze}

Computing details

Data collection: Marccd (Doyle, 2011); cell refinement: iMosflm (Battye et al., 2011); data reduction: iMosflm; program(s) used to solve structure: SHELXS97 (Sheldrick, 2008); program(s) used to refine structure: SHELXL2018 (Sheldrick, 2015); molecular graphics: ORTEP-3 for Windows (Farrugia, 2012); software used to prepare material for publication: PLATON (Spek, 2009).

(E)-1-(4-Chlorophenyl)-2-[2,2-dichloro-1-(4-fluorophenyl)ethenyl] $\backslash$ diazene

Crystal data

$\mathrm{C}_{14} \mathrm{H}_{8} \mathrm{Cl}_{3} \mathrm{FN}_{2}$

$M_{r}=329.57$

Monoclinic, $P 2_{1} / c$

$a=3.8617(8) \AA$

$b=24.249(5) \AA$

$c=14.724(3) \AA$

$\beta=94.30(3)^{\circ}$

$V=1374.9(5) \AA^{3}$

$Z=4$

\section{Data collection}

Rayonix SX165 CCD

diffractometer

/f scan

Absorption correction: multi-scan

(Scala; Evans, 2006)

$T_{\min }=0.840, T_{\max }=0.970$

20761 measured reflections

\section{Refinement}

Refinement on $F^{2}$

Least-squares matrix: full

$R\left[F^{2}>2 \sigma\left(F^{2}\right)\right]=0.053$

$w R\left(F^{2}\right)=0.142$

$S=1.05$

2984 reflections

182 parameters

0 restraints
$F(000)=664$

$D_{\mathrm{x}}=1.592 \mathrm{Mg} \mathrm{m}^{-3}$

Synchrotron radiation, $\lambda=0.80246 \AA$

Cell parameters from 600 reflections

$\theta=3.3-30.0^{\circ}$

$\mu=0.93 \mathrm{~mm}^{-1}$

$T=100 \mathrm{~K}$

Plate, orange

$0.20 \times 0.10 \times 0.02 \mathrm{~mm}$

2984 independent reflections

2719 reflections with $I>2 \sigma(I)$

$R_{\text {int }}=0.115$

$\theta_{\max }=30.9^{\circ}, \theta_{\min }=3.3^{\circ}$

$h=-4 \rightarrow 4$

$k=-30 \rightarrow 31$

$l=-18 \rightarrow 18$

Primary atom site location: difference Fourier map

Secondary atom site location: difference Fourier map

Hydrogen site location: inferred from neighbouring sites

$\mathrm{H}$-atom parameters constrained

$w=1 /\left[\sigma^{2}\left(F_{0}^{2}\right)+(0.0557 P)^{2}+1.092 P\right]$

where $P=\left(F_{\mathrm{o}}^{2}+2 F_{\mathrm{c}}{ }^{2}\right) / 3$ 
$(\Delta / \sigma)_{\max }=0.001$

$\Delta \rho_{\max }=0.59 \mathrm{e} \AA^{-3}$

$\Delta \rho_{\min }=-0.72$ e $\AA^{-3}$
Extinction correction: SHELXL2018

(Sheldrick, 2015),

$\mathrm{Fc}^{*}=\mathrm{kFc}\left[1+0.001 \mathrm{xFc}^{2} \lambda^{3} / \sin (2 \theta)\right]^{-1 / 4}$

Extinction coefficient: $0.026(3)$

\section{Special details}

Geometry. All esds (except the esd in the dihedral angle between two 1.s. planes) are estimated using the full covariance matrix. The cell esds are taken into account individually in the estimation of esds in distances, angles and torsion angles; correlations between esds in cell parameters are only used when they are defined by crystal symmetry. An approximate (isotropic) treatment of cell esds is used for estimating esds involving l.s. planes.

Fractional atomic coordinates and isotropic or equivalent isotropic displacement parameters $\left(\AA^{2}\right)$

\begin{tabular}{lllll}
\hline & $x$ & $y$ & $z$ & $U_{\text {iso }} / U_{\text {eq }}$ \\
\hline C11 & $0.68912(17)$ & $0.12097(2)$ & $0.17209(4)$ & $0.0265(2)$ \\
C12 & $0.48467(18)$ & $0.22728(2)$ & $0.10431(4)$ & $0.0283(2)$ \\
C13 & $-0.18247(18)$ & $0.50802(2)$ & $0.35787(5)$ & $0.0318(2)$ \\
F1 & $0.5868(5)$ & $0.06509(7)$ & $0.58998(10)$ & $0.0386(4)$ \\
N1 & $0.3562(6)$ & $0.25699(8)$ & $0.28387(14)$ & $0.0246(5)$ \\
N2 & $0.2435(6)$ & $0.27366(8)$ & $0.35685(14)$ & $0.0230(4)$ \\
C1 & $0.4622(7)$ & $0.20110(9)$ & $0.28183(16)$ & $0.0225(5)$ \\
C2 & $0.5361(7)$ & $0.18506(9)$ & $0.19760(16)$ & $0.0237(5)$ \\
C3 & $0.4936(7)$ & $0.16469(9)$ & $0.36354(16)$ & $0.0232(5)$ \\
C4 & $0.6716(7)$ & $0.18329(10)$ & $0.44378(16)$ & $0.0249(5)$ \\
H4 & 0.7714 & 0.2191 & 0.4459 & $0.030^{*}$ \\
C5 & $0.7036(7)$ & $0.14978(10)$ & $0.52038(16)$ & $0.0283(6)$ \\
H5 & 0.8242 & 0.1622 & 0.5752 & $0.034^{*}$ \\
C6 & $0.5558(8)$ & $0.09804(10)$ & $0.51483(17)$ & $0.0287(6)$ \\
C7 & $0.3803(7)$ & $0.07791(10)$ & $0.43694(17)$ & $0.0280(5)$ \\
H7 & 0.2840 & 0.0418 & 0.4352 & $0.034^{*}$ \\
C8 & $0.3485(7)$ & $0.11196(10)$ & $0.36103(17)$ & $0.0243(5)$ \\
H8 & 0.2264 & 0.0992 & 0.3067 & $0.029^{*}$ \\
C9 & $0.1482(7)$ & $0.33066(9)$ & $0.35229(16)$ & $0.0225(5)$ \\
C10 & $0.1990(7)$ & $0.36475(10)$ & $0.27784(16)$ & $0.0251(5)$ \\
H10 & 0.3012 & 0.3504 & 0.2261 & $0.030^{*}$ \\
C11 & $0.1000(7)$ & $0.41943(10)$ & $0.27997(16)$ & $0.0257(5)$ \\
H11 & 0.1332 & 0.4430 & 0.2298 & $0.031^{*}$ \\
C12 & $-0.0490(7)$ & $0.43956(10)$ & $0.35640(17)$ & $0.0246(5)$ \\
C13 & $-0.0997(7)$ & $0.40658(10)$ & $0.43064(17)$ & $0.0255(5)$ \\
H13 & -0.2002 & 0.4212 & 0.4824 & $0.031^{*}$ \\
C14 & $-0.0012(7)$ & $0.35157(10)$ & $0.42818(16)$ & $0.0248(5)$ \\
H14 & -0.0358 & 0.3282 & 0.4784 & $0.030^{*}$ \\
& & & &
\end{tabular}

Atomic displacement parameters $\left(\AA^{2}\right)$

\begin{tabular}{lllllll}
\hline & $U^{11}$ & $U^{22}$ & $U^{33}$ & $U^{12}$ & $U^{13}$ & $U^{23}$ \\
\hline C11 & $0.0363(4)$ & $0.0157(3)$ & $0.0273(3)$ & $0.0018(2)$ & $0.0006(2)$ & $-0.0038(2)$ \\
C12 & $0.0408(4)$ & $0.0196(3)$ & $0.0243(3)$ & $0.0022(2)$ & $0.0017(2)$ & $0.0020(2)$ \\
C13 & $0.0384(4)$ & $0.0125(3)$ & $0.0442(4)$ & $0.0020(2)$ & $0.0011(3)$ & $0.0002(2)$
\end{tabular}




\begin{tabular}{lllllll} 
F1 & $0.0622(12)$ & $0.0241(8)$ & $0.0290(8)$ & $0.0040(8)$ & $-0.0004(7)$ & $0.0087(6)$ \\
N1 & $0.0333(12)$ & $0.0135(9)$ & $0.0266(10)$ & $-0.0008(8)$ & $0.0000(8)$ & $-0.0019(7)$ \\
N2 & $0.0292(11)$ & $0.0128(9)$ & $0.0269(10)$ & $0.0004(8)$ & $0.0008(8)$ & $-0.0015(7)$ \\
C1 & $0.0273(13)$ & $0.0123(10)$ & $0.0272(11)$ & $-0.0027(9)$ & $-0.0017(9)$ & $-0.0010(8)$ \\
C2 & $0.0286(13)$ & $0.0146(10)$ & $0.0272(11)$ & $-0.0032(9)$ & $-0.0019(9)$ & $-0.0014(8)$ \\
C3 & $0.0301(13)$ & $0.0143(11)$ & $0.0253(11)$ & $0.0017(9)$ & $0.0013(9)$ & $-0.0013(8)$ \\
C4 & $0.0316(14)$ & $0.0149(11)$ & $0.0279(11)$ & $0.0016(9)$ & $0.0007(10)$ & $-0.0005(9)$ \\
C5 & $0.0361(15)$ & $0.0214(12)$ & $0.0267(11)$ & $0.0039(10)$ & $-0.0023(10)$ & $-0.0023(9)$ \\
C6 & $0.0407(15)$ & $0.0175(11)$ & $0.0280(11)$ & $0.0064(10)$ & $0.0037(10)$ & $0.0060(9)$ \\
C7 & $0.0376(15)$ & $0.0143(11)$ & $0.0321(12)$ & $0.0007(10)$ & $0.0037(10)$ & $0.0016(9)$ \\
C8 & $0.0291(13)$ & $0.0153(11)$ & $0.0285(11)$ & $0.0000(9)$ & $0.0013(10)$ & $-0.0017(9)$ \\
C9 & $0.0288(13)$ & $0.0112(10)$ & $0.0269(11)$ & $0.0003(9)$ & $-0.0025(9)$ & $-0.0011(8)$ \\
C10 & $0.0315(14)$ & $0.0176(11)$ & $0.0259(11)$ & $-0.0001(9)$ & $-0.0004(9)$ & $0.0001(9)$ \\
C11 & $0.0332(14)$ & $0.0157(11)$ & $0.0277(11)$ & $-0.0010(9)$ & $-0.0020(10)$ & $0.0022(9)$ \\
C12 & $0.0286(13)$ & $0.0132(11)$ & $0.0312(12)$ & $-0.0020(9)$ & $-0.0037(10)$ & $-0.0005(9)$ \\
C13 & $0.0302(13)$ & $0.0165(11)$ & $0.0292(11)$ & $-0.0012(9)$ & $-0.0009(9)$ & $-0.0037(9)$ \\
C14 & $0.0319(14)$ & $0.0175(11)$ & $0.0243(11)$ & $-0.0007(9)$ & $-0.0019(9)$ & $0.0013(9)$ \\
& & & & & & \\
\hline
\end{tabular}

Geometric parameters $\left(\AA,{ }^{\circ}\right)$

\begin{tabular}{llll}
\hline $\mathrm{C} 11-\mathrm{C} 2$ & $1.714(2)$ & $\mathrm{C} 6-\mathrm{C} 7$ & $1.377(4)$ \\
$\mathrm{C} 12-\mathrm{C} 2$ & $1.713(2)$ & $\mathrm{C} 7-\mathrm{C} 8$ & $1.388(3)$ \\
$\mathrm{C} 13-\mathrm{C} 12$ & $1.739(2)$ & $\mathrm{C} 7-\mathrm{H} 7$ & 0.9500 \\
$\mathrm{~F} 1-\mathrm{C} 6$ & $1.363(3)$ & $\mathrm{C} 8-\mathrm{H} 8$ & 0.9500 \\
$\mathrm{~N} 1-\mathrm{N} 2$ & $1.256(3)$ & $\mathrm{C} 9-\mathrm{C} 14$ & $1.391(3)$ \\
$\mathrm{N} 1-\mathrm{C} 1$ & $1.417(3)$ & $\mathrm{C} 9-\mathrm{C} 10$ & $1.399(3)$ \\
$\mathrm{N} 2-\mathrm{C} 9$ & $1.431(3)$ & $\mathrm{C} 10-\mathrm{C} 11$ & 0.9500 \\
$\mathrm{C} 1-\mathrm{C} 2$ & $1.351(3)$ & $\mathrm{C} 10-\mathrm{H} 10$ & $1.390(4)$ \\
$\mathrm{C} 1-\mathrm{C} 3$ & $1.490(3)$ & $\mathrm{C} 11-\mathrm{C} 12$ & 0.9500 \\
$\mathrm{C} 3-\mathrm{C} 8$ & $1.395(3)$ & $\mathrm{C} 11-\mathrm{H} 11$ & $1.380(3)$ \\
$\mathrm{C} 3-\mathrm{C} 4$ & $1.396(3)$ & $\mathrm{C} 12-\mathrm{C} 13$ & $1.389(3)$ \\
$\mathrm{C} 4-\mathrm{C} 5$ & $1.388(3)$ & $\mathrm{C} 13-\mathrm{C} 14$ & 0.9500 \\
$\mathrm{C} 4-\mathrm{H} 4$ & 0.9500 & $\mathrm{C} 13-\mathrm{H} 13$ & 0.9500 \\
$\mathrm{C} 5-\mathrm{C} 6$ & $1.378(4)$ & $\mathrm{C} 14-\mathrm{H} 14$ & \\
$\mathrm{C} 5-\mathrm{H} 5$ & 0.9500 & & 121.0 \\
& & & $120.9(2)$ \\
$\mathrm{N} 2-\mathrm{N} 1-\mathrm{C} 1$ & $116.43(19)$ & $\mathrm{C} 8-\mathrm{C} 7-\mathrm{H} 7$ & 119.6 \\
$\mathrm{~N} 1-\mathrm{N} 2-\mathrm{C} 9$ & $112.0(2)$ & $\mathrm{C} 7-\mathrm{C} 8-\mathrm{C} 3$ & $120.4(2)$ \\
$\mathrm{C} 2-\mathrm{C} 1-\mathrm{N} 1$ & $112.1(2)$ & $\mathrm{C} 7-\mathrm{C} 8-\mathrm{H} 8$ & $115.8(2)$ \\
$\mathrm{C} 2-\mathrm{C} 1-\mathrm{C} 3$ & $124.1(2)$ & $\mathrm{C} 3-\mathrm{C} 8-\mathrm{H} 8$ & $123.9(2)$ \\
$\mathrm{N} 1-\mathrm{C} 1-\mathrm{C} 3$ & $123.8(2)$ & $\mathrm{C} 14-\mathrm{C} 9-\mathrm{C} 10$ & $119.6(2)$ \\
$\mathrm{C} 1-\mathrm{C} 2-\mathrm{C} 12$ & $122.99(19)$ & $\mathrm{C} 14-\mathrm{C} 9-\mathrm{N} 2$ & 120.2 \\
$\mathrm{C} 1-\mathrm{C} 2-\mathrm{C} 11$ & $124.22(18)$ & $\mathrm{C} 10-\mathrm{C} 9-\mathrm{N} 2$ & 120.2 \\
$\mathrm{C} 12-\mathrm{C} 2-\mathrm{C} 11$ & $112.79(14)$ & $\mathrm{C} 11-\mathrm{C} 10-\mathrm{C} 9$ & $119.2(2)$ \\
$\mathrm{C} 8-\mathrm{C} 3-\mathrm{C} 4$ & $119.3(2)$ & $\mathrm{C} 11-\mathrm{C} 10-\mathrm{H} 10$ & 120.4 \\
$\mathrm{C} 8-\mathrm{C} 3-\mathrm{C} 1$ & $120.9(2)$ & $\mathrm{C} 9-\mathrm{C} 10-\mathrm{H} 10$ & $\mathrm{C} 10-\mathrm{C} 11-\mathrm{C} 12$ \\
$\mathrm{C} 4-\mathrm{C} 3-\mathrm{C} 1$ & $119.8(2)$ & $\mathrm{C} 10-\mathrm{C} 11-\mathrm{H} 11$ & \\
$\mathrm{C} 5-\mathrm{C} 4-\mathrm{C} 3$ & $120.4(2)$ & &
\end{tabular}




\begin{tabular}{|c|c|c|c|}
\hline $\mathrm{C} 5-\mathrm{C} 4-\mathrm{H} 4$ & 119.8 & $\mathrm{C} 12-\mathrm{C} 11-\mathrm{H} 11$ & 120.4 \\
\hline $\mathrm{C} 3-\mathrm{C} 4-\mathrm{H} 4$ & 119.8 & $\mathrm{C} 13-\mathrm{C} 12-\mathrm{C} 11$ & $122.0(2)$ \\
\hline $\mathrm{C} 6-\mathrm{C} 5-\mathrm{C} 4$ & $118.3(2)$ & $\mathrm{C} 13-\mathrm{C} 12-\mathrm{Cl} 3$ & $118.9(2)$ \\
\hline $\mathrm{C} 6-\mathrm{C} 5-\mathrm{H} 5$ & 120.9 & $\mathrm{C} 11-\mathrm{C} 12-\mathrm{Cl} 3$ & $119.10(18)$ \\
\hline $\mathrm{C} 4-\mathrm{C} 5-\mathrm{H} 5$ & 120.9 & $\mathrm{C} 12-\mathrm{C} 13-\mathrm{C} 14$ & $118.7(2)$ \\
\hline $\mathrm{F} 1-\mathrm{C} 6-\mathrm{C} 7$ & $118.4(2)$ & $\mathrm{C} 12-\mathrm{C} 13-\mathrm{H} 13$ & 120.6 \\
\hline $\mathrm{F} 1-\mathrm{C} 6-\mathrm{C} 5$ & $118.3(2)$ & $\mathrm{C} 14-\mathrm{C} 13-\mathrm{H} 13$ & 120.6 \\
\hline $\mathrm{C} 7-\mathrm{C} 6-\mathrm{C} 5$ & $123.2(2)$ & $\mathrm{C} 13-\mathrm{C} 14-\mathrm{C} 9$ & $120.1(2)$ \\
\hline $\mathrm{C} 6-\mathrm{C} 7-\mathrm{C} 8$ & $117.9(2)$ & $\mathrm{C} 13-\mathrm{C} 14-\mathrm{H} 14$ & 119.9 \\
\hline $\mathrm{C} 6-\mathrm{C} 7-\mathrm{H} 7$ & 121.0 & C9-C14-H14 & 119.9 \\
\hline $\mathrm{C} 1-\mathrm{N} 1-\mathrm{N} 2-\mathrm{C} 9$ & $179.2(2)$ & $\mathrm{C} 5-\mathrm{C} 6-\mathrm{C} 7-\mathrm{C} 8$ & $-0.8(4)$ \\
\hline $\mathrm{N} 2-\mathrm{N} 1-\mathrm{C} 1-\mathrm{C} 2$ & $171.9(2)$ & $\mathrm{C} 6-\mathrm{C} 7-\mathrm{C} 8-\mathrm{C} 3$ & $0.7(4)$ \\
\hline $\mathrm{N} 2-\mathrm{N} 1-\mathrm{C} 1-\mathrm{C} 3$ & $-9.0(4)$ & $\mathrm{C} 4-\mathrm{C} 3-\mathrm{C} 8-\mathrm{C} 7$ & $-0.3(4)$ \\
\hline $\mathrm{N} 1-\mathrm{C} 1-\mathrm{C} 2-\mathrm{Cl} 2$ & $-3.0(3)$ & $\mathrm{C} 1-\mathrm{C} 3-\mathrm{C} 8-\mathrm{C} 7$ & $179.5(2)$ \\
\hline $\mathrm{C} 3-\mathrm{C} 1-\mathrm{C} 2-\mathrm{Cl} 2$ & $177.94(19)$ & $\mathrm{N} 1-\mathrm{N} 2-\mathrm{C} 9-\mathrm{C} 14$ & $175.9(2)$ \\
\hline $\mathrm{N} 1-\mathrm{C} 1-\mathrm{C} 2-\mathrm{C} 11$ & $177.14(18)$ & $\mathrm{N} 1-\mathrm{N} 2-\mathrm{C} 9-\mathrm{C} 10$ & $-4.5(4)$ \\
\hline $\mathrm{C} 3-\mathrm{C} 1-\mathrm{C} 2-\mathrm{Cl} 1$ & $-1.9(4)$ & $\mathrm{C} 14-\mathrm{C} 9-\mathrm{C} 10-\mathrm{C} 11$ & $0.0(4)$ \\
\hline $\mathrm{C} 2-\mathrm{C} 1-\mathrm{C} 3-\mathrm{C} 8$ & $-49.2(4)$ & $\mathrm{N} 2-\mathrm{C} 9-\mathrm{C} 10-\mathrm{C} 11$ & $-179.6(2)$ \\
\hline $\mathrm{N} 1-\mathrm{C} 1-\mathrm{C} 3-\mathrm{C} 8$ & $131.8(3)$ & $\mathrm{C} 9-\mathrm{C} 10-\mathrm{C} 11-\mathrm{C} 12$ & $0.0(4)$ \\
\hline $\mathrm{C} 2-\mathrm{C} 1-\mathrm{C} 3-\mathrm{C} 4$ & $130.5(3)$ & $\mathrm{C} 10-\mathrm{C} 11-\mathrm{C} 12-\mathrm{C} 13$ & $0.3(4)$ \\
\hline $\mathrm{N} 1-\mathrm{C} 1-\mathrm{C} 3-\mathrm{C} 4$ & $-48.4(4)$ & $\mathrm{C} 10-\mathrm{C} 11-\mathrm{C} 12-\mathrm{Cl} 3$ & $-178.57(19)$ \\
\hline $\mathrm{C} 8-\mathrm{C} 3-\mathrm{C} 4-\mathrm{C} 5$ & $-0.1(4)$ & $\mathrm{C} 11-\mathrm{C} 12-\mathrm{C} 13-\mathrm{C} 14$ & $-0.6(4)$ \\
\hline $\mathrm{C} 1-\mathrm{C} 3-\mathrm{C} 4-\mathrm{C} 5$ & $-179.9(2)$ & $\mathrm{Cl} 3-\mathrm{C} 12-\mathrm{C} 13-\mathrm{C} 14$ & $178.31(19)$ \\
\hline $\mathrm{C} 3-\mathrm{C} 4-\mathrm{C} 5-\mathrm{C} 6$ & $0.0(4)$ & $\mathrm{C} 12-\mathrm{C} 13-\mathrm{C} 14-\mathrm{C} 9$ & $0.5(4)$ \\
\hline $\mathrm{C} 4-\mathrm{C} 5-\mathrm{C} 6-\mathrm{F} 1$ & $-180.0(2)$ & $\mathrm{C} 10-\mathrm{C} 9-\mathrm{C} 14-\mathrm{C} 13$ & $-0.2(4)$ \\
\hline $\mathrm{C} 4-\mathrm{C} 5-\mathrm{C} 6-\mathrm{C} 7$ & $0.4(4)$ & $\mathrm{N} 2-\mathrm{C} 9-\mathrm{C} 14-\mathrm{C} 13$ & $179.4(2)$ \\
\hline $\mathrm{F} 1-\mathrm{C} 6-\mathrm{C} 7-\mathrm{C} 8$ & $179.6(2)$ & & \\
\hline
\end{tabular}

Hydrogen-bond geometry $\left(A,{ }^{\circ}\right)$

\begin{tabular}{lllll}
\hline$D-\mathrm{H} \cdots A$ & $D-\mathrm{H}$ & $\mathrm{H} \cdots A$ & $D \cdots A$ & $D-\mathrm{H} \cdots A$ \\
\hline $\mathrm{C} 8-\mathrm{H} 8 \cdots \mathrm{C} 11^{\mathrm{i}}$ & 0.95 & 2.81 & $3.634(3)$ & 146 \\
\hline
\end{tabular}

Symmetry code: (i) $x-1, y, z$. 